\title{
BMJ Open Maternal reproductive history and premenopausal risk of hypertension and cardiovascular disease: a Danish cohort study
}

\author{
Shannon X Chen, ${ }^{1}$ Kathleen M Rasmussen, ${ }^{1}$ Julia Finkelstein, ${ }^{1} \mathrm{H}$ Støvring, ${ }^{2}$ \\ Ellen Aa Nøhr, ${ }^{3}$ Helene Kirkegaard ${ }^{3}$
}

To cite: Chen SX,

Rasmussen KM, Finkelstein J, et al. Maternal reproductive history and premenopausal risk of hypertension and cardiovascular disease: a Danish cohort study. BMJ Open 2019;9:e030702. doi:10.1136/ bmjopen-2019-030702

- Prepublication history and additional material for this paper are available online. To view these files, please visit the journal online (http://dx.doi. org/10.1136/bmjopen-2019030702).

Received 02 April 2019 Revised 23 August 2019 Accepted 12 September 2019

Check for updates

C Author(s) (or their employer(s)) 2019. Re-use permitted under CC BY-NC. No commercial re-use. See rights and permissions. Published by BMJ.

${ }^{1}$ Division of Nutritional Sciences, Cornell University, Ithaca, New York, USA

${ }^{2}$ Department of Public Health, Section for Biostatistics, Aarhus University, Aarhus, Denmark ${ }^{3}$ Department of Clinical Research, the Research Unit of Gynecology and Obstetrics, University of Southern Denmark, Odense, Denmark

Correspondence to

Dr Helene Kirkegaard;

hkirkegaard@health.sdu.dk

\section{ABSTRACT}

Objectives The metabolic changes of pregnancy resemble a cardiovascular risk profile and may persist postpartum, with body mass index (BMI) as a potential modifier. We examined the association between the number of livebirth pregnancies and maternal premenopausal risks of hypertension and cardiovascular disease (CVD), accounting for pre-pregnancy BMl as well as abortions and stillbirths. Design A prospective cohort study.

Setting Mothers from the Danish National Birth Cohort (1996 to 2002). For each of these women, registry data on all pregnancies from 1973 to 2011 were obtained, as were self-reported pre-pregnancy weight and height.

Participants A total of 44552 first-time mothers in the Danish National Birth Cohort.

Outcome measures Risks of hypertension and CVD during and between live-birth pregnancies separately and combined as live-birth cycles.

Results After adjustment for abortions, stillbirths, prepregnancy BMI and other covariates, a higher risk of hypertension was observed in the first (HR 1.53, 95\% $\mathrm{Cl}: 1.37$ to 1.72 ) and fourth and subsequent live-birth cycles (HR 1.72, 95\% Cl: 1.15 to 2.58), compared with the second. However, as number of live-birth pregnancies increased, risk of hypertension decreased during live-birth pregnancies and increased between live-birth pregnancies (tests for trend, $p<0.01$ ). For CVD, we found an overall $\mathrm{J}$-shaped but non-significant association with number of live-birth pregnancies. No interaction with pre-pregnancy BMI $\left(<25\right.$ versus $\left.\geq 25 \mathrm{~kg} / \mathrm{m}^{2}\right)$ was observed.

Conclusions Premenopausal women had the highest risk of hypertension and CVD during their first live-birth pregnancy and after their fourth live-birth pregnancy. All risks were independent of BMI before the first live-birth pregnancy and of number of abortions and stillbirths.

\section{INTRODUCTION}

Cardiovascular disease (CVD) is the leading cause of death worldwide, with obesity and hypertension as important risk factors. ${ }^{1}$ Women experience significant metabolic changes during pregnancy, including increased fat mass, blood glucose, triglyceride and low-density lipoprotein cholesterol concentrations. ${ }^{2}$ Although these changes
Strengths and limitations of this study

- The combination of cohort and register data limited risk of confounding by behavioural factors and selection bias due to loss to follow-up.

- An extensive register-based reproductive record was developed for each woman to adjust for pregnancy losses, including induced and spontaneous abortions.

- We studied premenopausal risks of hypertension and cardiovascular disease and differentiated risk during live-birth pregnancies from risk between live-birth pregnancies.

- Maternal body mass index at start of the reproductive period was considered as a potential modifier.

- Although maternal socio-occupational status and lifestyle may change over the reproductive period, it was only available at first live-birth pregnancy.

during pregnancy are largely reversed after delivery, this inflammatory state may still increase the risk of hypertension and CVD later in life. ${ }^{3}{ }^{4}$ Previous research on the associations between parity and hypertension and CVD has not been consistent. Most researchers have studied postmenopausal women and reported an increased risk of CVD with parity or a J-shaped association with two births as the nadir of risk..$^{5-11}$ Premenopausal risk of CVD and hypertension has only been examined in few studies that show inconclusive findings. ${ }^{12} 13$ None of these studies followed women through their reproductive period, considered the potential different risks during and between pregnancies or accounted for the mother's history of pregnancy loss, even though the metabolic changes that resemble a cardiovascular risk profile occur in all pregnancies, also when the pregnancy does not result in a live birth.

Obesity is a risk factor for hypertension $^{14}$ and CVD. ${ }^{1}$ During pregnancy, obese women have increased insulin resistance, 


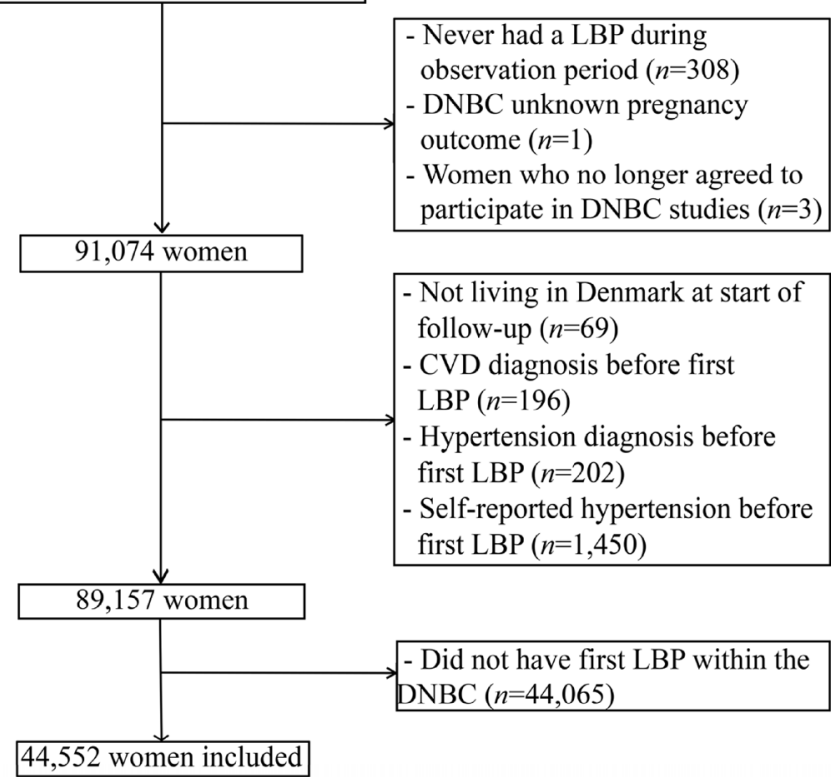

Figure 1 Participant flow chart of women in the Danish National Birth Cohort (DNBC), 1996 to 2002, with inclusion and exclusion criteria for live-birth pregnancies (LBPs) and hypertension and cardiovascular disease (CVD) diagnoses.

inflammatory biomarkers, triglycerides and low-density lipoprotein cholesterol concentrations, compared with normal-weight or underweight women. ${ }^{15}$ Therefore, prepregnancy obesity may amplify the potential association between pregnancy and risk of hypertension and CVD.

The objective of this study was to examine the associations between the number of live-birth pregnancies (LBPs) and maternal premenopausal risk of hypertension and CVD, while accounting for pregnancy loss. Our first hypothesis was a J-shaped association between the number of LBPs and risk of hypertension and CVD, with the lowest risk with two LBPs. Our second hypothesis was that this association would be stronger among women who were overweight/obese before their first LBP compared with women who were normal-weight/underweight.

\section{METHODS}

\section{Study population}

This prospective study was conducted among participants in the Danish National Birth Cohort (DNBC), which includes extensive information on 100418 pregnancies of 91386 women recruited between 1996 and 2002. ${ }^{16}$ Selfreported pregnancy-related information was obtained in four telephone interviews at approximately 16 and 30 weeks of gestation, and at 6 and 18 months postpartum. ${ }^{16}$ We excluded women from the analyses for the following reasons: withdrew from the DNBC $(n=3)$, no LBP in the follow-up period $(n=308)$, unknown outcome of a DNBC pregnancy $(n=1)$, emigrated before start of follow-up $(\mathrm{n}=69)$, diagnosed with hypertension and/or CVD at the hospital before their first LBP $(\mathrm{n}=398)$ (see online supplementary table 1 for the International Classification of Disease (ICD) codes), and self-reported hypertension before their first LBP $(n=1450)$. Data on self-reported hypertension before the first LBP was obtained from the first pregnancy interview. Finally, inasmuch as women with a prior pregnancy-related CVD diagnosis at the time of DNBC enrolment may be less likely to be pregnant again and therefore not eligible for enrolment in the DNBC, we excluded 44605 women who were parous at time of DNBC enrolment to prevent potential selection problems and period effects. Our final study population included 44552 women (figure 1). The Scientific Ethics Committee in Denmark and the Danish Data Protection Agency approved the DNBC and the latter also the present sub-study. All the participants in the DNBC provided written informed consent.

\section{Patient and public involvement}

Patients and public were not involved in the present study.

\section{Reproductive history}

We established a full reproductive history for each participant (1973 to 2011) by linkage to registry data using the unique identification numbers from the Danish Civil Registration System (see online supplementary figure 1 for timeline). Because we only included women who had their first LBP within the DNBC, all LBPs occurred between 1996 to 2011. Data on births not recorded in the DNBC were extracted from the Medical Birth Registry (MBR).${ }^{17}$ Because of a shift in the definition of a stillbirth recorded in the registers, a stillbirth was defined as fetal demise that occurred at $\geq 28$ weeks gestation from 1973 to 2003 and at $\geq 22$ weeks gestation after $2003 .{ }^{18}$ Data for spontaneous abortions (miscarriages) were obtained from the National Patient Registry (NPR) (1977 to 2011).${ }^{19}$ Induced abortions were recorded in the Registry of Legally Induced Abortions (1974 to 1994) ${ }^{20}$ and the NPR (1995 to 2011). See online supplementary table 1 for specific codes included. In total, we identified 289372 pregnancies for our data set.

Our exposure was number of LBPs as we wanted to account for number of complete reproductive cycles, which included a full pregnancy, a potential period of breastfeeding and child-rearing-related behavioural changes. Thus, stillbirths were not included in the exposure, but were adjusted for as done for spontaneous and induced abortions.

\section{Outcomes}

Hypertension and CVD diagnostic ICD-8 and ICD-10 codes were extracted from the NPR from January 1977 to February 2016. The NPR contains information on all inpatient contacts since 1977 and also outpatient contacts since $1995 .{ }^{19} \mathrm{CVD}$ outcomes in this study included incident diagnoses of ischaemic heart disease, stroke, atherosclerosis and thrombosis, and pregnancy-specific diagnoses of thrombosis and stroke. Hypertension outcomes in this study included both chronic hypertension and gestational hypertension (see online supplementary table 1 
for specific ICD codes and online supplementary figure 1 for a timeline of the data sources). We did not include preeclampsia as it may have a different origin/aetiology than hypertension and CVD. We also did not adjust for preeclampsia because it is a risk factor for later hypertension and CVD and therefore a potential intermediator on the pathway from LBPs to hypertension and CVD.

\section{Covariates}

In the first DNBC pregnancy interview, women provided information about their weight $(\mathrm{kg})$ and height $(\mathrm{m})$, alcohol intake before pregnancy ( 0,1 to $<7$ or $\geq 7$ units/ week) and leisure-time exercise during pregnancy $(0,1$ to 180 or $>180 \mathrm{~min} /$ week). Body mass index (BMI) $\left(\mathrm{kg} / \mathrm{m}^{2}\right)$ was categorised using WHO criteria $(<18.5,18.5$ to $<25,25$ to $<30, \geq 30 \mathrm{~kg} / \mathrm{m}^{2}$ ). Underweight/normal-weight women included those with BMI $<25 \mathrm{~kg} / \mathrm{m}^{2}$ and overweight/ obese women included those with BMI $\geq 25 \mathrm{~kg} / \mathrm{m}^{2}$. Information on socio-occupational status was also obtained from the first pregnancy interview and categorised based on occupation type and education level (high, middle, low). ${ }^{21}$ Smoking status was obtained from the MBR beginning in 1991 and defined as a fixed dichotomous variable (ever/never smoked during any pregnancy).

\section{Statistical analyses}

All statistical analyses were conducted using Stata V.15.1 (StataCorp, College Station, Texas). Pearson $\chi^{2}$ tests were used to compare maternal characteristics across total number of LBPs during follow-up.

We conducted Cox proportional hazard models to examine premenopausal incident hypertension and CVD during three time periods: (a) live-birth cycles (LBCs), (b) during LBPs and (c) between (inter) LBPs (figure 2). An LBC was defined as the time from one LBP-conception to the next, or to the end of follow-up if there were no subsequent LBPs. Our exposure (LBPs) was time-dependent; thus, women changed exposure group at the conception of each new LBP. We used maternal age as the underlying time axis and women provided risk time from conception (ie, the first day of the last menstrual period) of their first LBP, which had to be within the DNBC enrolment period (1996 to 2002). Follow-up continued until the time of hypertension or CVD diagnosis, emigration, death or end of follow-up, whichever came first. The end of follow-up for the during-LBP and inter-LBP analyses was July 2011, when our data on reproductive histories ended. The end of follow-up for the LBC analyses was February 2016, with women keeping their 2011 exposure status through the end of follow-up, as most women would not have more births after that time. A diagnosis of hypertension or CVD that occurred within the interval from conception to 42 days postpartum was considered to have occurred during a LBP, in accord with the WHO definition of a maternal death. ${ }^{22}$ A diagnosis of hypertension or CVD that occurred after 42 days postpartum and before the next conception was considered to have occurred in an inter-LBP interval. In analyses of risk during LBPs, we adjusted for prior hypertension or CVD diagnosis in all past inter-LBP intervals. Likewise, in the analyses of risk in inter-LBP intervals, we adjusted for prior hypertension or CVD diagnosis in all past LBP intervals. We did this to focus on the first occurrence of a diagnosis in the period of interest, but still account for potential confounding by a prior diagnosis in the other period. In the analyses of LBCs, women were only considered at risk until their first diagnosis of hypertension or CVD and then censored. We created graphical evaluations of the assumption of proportional hazards and did not observe any violation.

All models were analysed with maternal age as the underlying time-scale (Model 1). We further adjusted for a priori-selected covariates by including induced abortions, spontaneous abortions and stillbirths as time-dependent

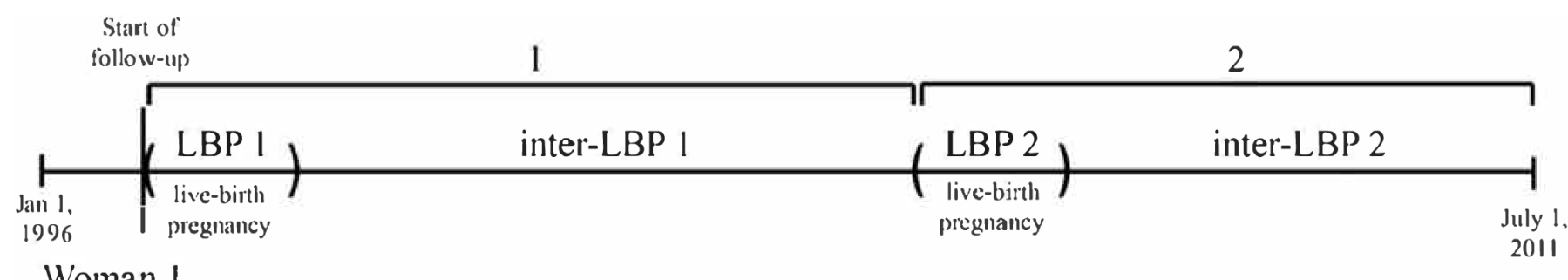

Woman 1

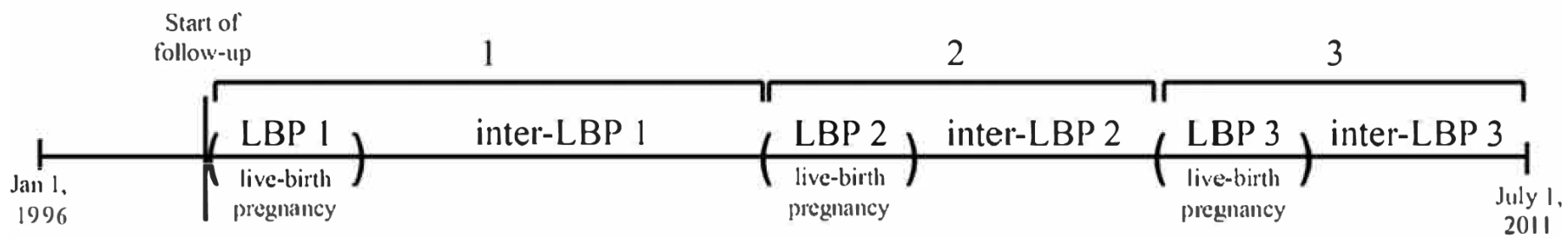

Woman 2

Figure 2 Diagram presenting the follow-up period indicating each live-birth cycle, including live-birth pregnancy (LBP) and inter-live-birth pregnancy (inter-LBP) intervals of two women with two or three LBPs during the follow-up period, respectively. 
covariates as they occurred (Model 2), and smoking status, socio-occupational status, leisure-time exercise during pregnancy, alcohol intake before pregnancy and pre-pregnancy BMI category, all as fixed variables (Model 3 ), thus these were adjusted for throughout the follow-up period. Analyses of Model 1 and Model 2 were based on the whole study population $(\mathrm{n}=44552)$ while the analyses of Model 3 were reduced slightly to 40177 women due to missing data on covariates.

We considered pre-pregnancy BMI as a potential modifier of the association between the number of LBPs and premenopausal risk of hypertension and CVD. We stratified the analyses on pre-pregnancy BMI category and tested for interactions with pre-pregnancy BMI category.

Finally, we conducted sensitivity analyses of the associations between number of LBPs and risk of hypertension and CVD among women who were parous at DNBC enrolment and had been excluded due to potential selection problems. As with the main analysis, follow-up started at conception of their first LBP; however, this means that follow-up may have started before 1996 as these women were parous at enrolment in the DNBC. We also did a sensitivity analysis excluding the $5 \%$ of the women who were above 51 years of age at the end of follow-up from the study population because they may be postmenopausal.

\section{RESULTS}

Among the study population, 19\% had one LBP, 57\% two LBPs, $21 \%$ three LBPs and 3\% four or more LBPs. Compared with women who only had one LBP, women who had more LBPs were younger at birth of their first LBP and had a higher total number of spontaneous abortions. As number of total LBPs increased from one to three, more women had no induced abortions, were of higher socio-occupational status, engaged in leisuretime exercise during pregnancy and were less likely to smoke during pregnancy or be overweight/obese before first LBP (table 1). At the end of follow-up, the median age was 43 years (IQR: 41 to 46). The median follow-up time was 11.3 years (IQR: 10.1 to 12.5 ) for during-LBP/ inter-LBP analyses and 15.8 years (IQR: 14.5 to 17.1 ) for LBC analyses.

\section{Hypertension}

A total of 1731 diagnoses of incident hypertension were recorded during follow-up (table 2). We found a J-shaped association between an increased number of LBCs and risk of hypertension that was similar after adjustment for abortions and stillbirths (Model 2) and in the fully adjusted model (Model 3). Thus, compared with the second LBC, we observed a $53 \%$ increased risk of hypertension in the first LBC (HR 1.53, 95\% CI: 1.37 to 1.72 ) and a $72 \%$ increased risk in the fourth and subsequent LBC (HR 1.72, 95\% CI: 1.15 to 2.58).

A total of 955 and 394 hypertension diagnoses were recorded during LBPs and between LBPs, respectively (table 2). Risk of hypertension during the first LBP was twice as high as risk during the second LBP (HR 2.10, 95\% CI: 1.79 to 2.47) and the risk further decreased as number of LBPs increased (test for trend, $\mathrm{p}<0.001$ ). In contrast, a $24 \%$ lower risk of hypertension was observed in the first inter-LBP compared with the second inter-LBP (HR 0.76, 95\% CI: 0.60 to 0.97 ) and the risk of hypertension further increased with greater number of LBPs (test for trend, $\mathrm{p}=0.004$ ). Adjustments only changed these associations slightly.

We observed no interaction $(\mathrm{p}=0.18)$ by pre-pregnancy BMI in the analyses of LBCs. Both normal/underweight women and overweight/obese women had an increased risk of hypertension in the first LBC (HR 1.63, 95\% CI: 1.40 to 1.89 and HR $1.45,95 \%$ CI: 1.23 to 1.71 , respectively) compared with the second LBC (table 2). However, only normal/underweight women had an increased risk of hypertension in the fourth and subsequent LBC compared with the second. The risks during pregnancies were modified by pre-pregnancy BMI, with a more clear decreasing trend observed in overweight/obese women than in normal/underweight women $(\mathrm{p}=0.04)$. No interaction by pre-pregnancy BMI was observed for risk of hypertension between LBPs $(\mathrm{p}=0.78)$.

\section{Cardiovascular disease}

There were 1147 CVD diagnoses reported during follow-up (table 3). We found a tendency towards a $\mathrm{J}$-shaped association with the lowest risk within the third LBC. Compared with the second LBC, a non-significant slightly increased risk of CVD was observed in the first LBC (HR 1.08, 95\% CI: 0.94 to 1.24) and fourth and subsequent LBC (HR 1.31, 95\% CI: 0.84 to 2.05 ).

A total of 146 and 537 CVD diagnoses were reported during a LBP and between LBPs, respectively (table 3). The few CVD cases during LBPs made the estimates uncertain, and none was statistically significant. In inter-LBP intervals, the trend in the estimates was similar to that of hypertension, but these were not statistically significant.

We observed no interaction by pre-pregnancy BMI in the analyses of LBCs, the analyses of CVD during LBPs or between LBPs with increasing number of LBPs $(\mathrm{p}=0.37$, $\mathrm{p}=0.70$ and $\mathrm{p}=0.27$, respectively) (table 3 ).

In sensitivity analyses among parous women at the time of enrolment into the DNBC, we observed a linear association between number of LBCs and risk of hypertension and CVD (online supplementary tables 2 and 3 ) in contrast to the J-shaped association we observed in our study sample. This suggests that if we had included women who were parous at the time of enrolment into the DNBC, it might have introduced selection bias. Another sensitivity analysis excluding women who were potential postmenopausal during follow-up showed similar results as those presented.

\section{DISCUSSION}

In this study, we prospectively examined premenopausal risks of hypertension and CVD throughout a woman's reproductive period and observed an overall J-shaped 
Table 1 Characteristics of 44552 women by total number of live-birth pregnancies during follow-up (1996 to 2011) participating in the Danish National Birth Cohort

\begin{tabular}{|c|c|c|c|c|c|}
\hline \multirow[b]{2}{*}{ Covariates } & \multicolumn{4}{|c|}{ Number of live-birth pregnancies } & \multirow[b]{2}{*}{$P$ value } \\
\hline & $1(n=8374)$ & $2(n=25368)$ & $3(n=9638)$ & $\geq 4(n=1172)$ & \\
\hline Age at first live-birth, y ( $n=44$ 552) & & & & & $<0.001$ \\
\hline$<25(\mathrm{n}=9677)$ & $1133(14)$ & $5075(20)$ & $2970(31)$ & $499(43)$ & \\
\hline $25-29(n=22350)$ & 2836 (34) & $13542(54)$ & $5415(56)$ & $557(48)$ & \\
\hline$\geq 30(n=12525)$ & $4405(53)$ & $6751(27)$ & $1253(13)$ & $116(10)$ & \\
\hline Number of induced abortions $(n=44552)$ & & & & & $<0.001$ \\
\hline $0(n=33697)$ & $5917(71)$ & $19390(76)$ & $7526(78)$ & $864(74)$ & \\
\hline$\geq 1(n=10855)$ & $2457(29)$ & $5978(24)$ & $2112(22)$ & $308(26)$ & \\
\hline Number of spontaneous abortions ( $n=44552$ ) & & & & & $<0.001$ \\
\hline $0(n=34964)$ & $6802(81)$ & $20277(80)$ & 7085 (74) & $800(68)$ & \\
\hline$\geq 1(n=9588)$ & $1572(19)$ & $5091(20)$ & $2553(26)$ & $372(32)$ & \\
\hline Number of stillbirths $(n=44552)$ & & & & & $<0.001$ \\
\hline $0(n=44268)$ & 8315 (99) & $25228(99)$ & 9562 (99) & $1163(99)$ & \\
\hline$\geq 1(n=284)$ & $59(1)$ & $140(1)$ & $76(1)$ & $9(1)$ & \\
\hline Preterm birth $(n=44552)$ & & & & & $<0.001$ \\
\hline Ever occurred $(n=4436)$ & $962(11)$ & $2345(9)$ & $985(10)$ & $144(12)$ & \\
\hline Never occurred $(n=40116)$ & $7412(89)$ & $23023(91)$ & $8653(90)$ & $1028(88)$ & \\
\hline Multiple birth $(n=44552)$ & & & & & $<0.001$ \\
\hline Ever had $(n=3664)$ & $758(9)$ & $1030(4)$ & $262(2)$ & $43(4)$ & \\
\hline Never had $(n=42459)$ & $7616(91)$ & $24338(96)$ & $9376(97)$ & 1129 (96) & \\
\hline Smoking during pregnancy $(n=44139)$ & & & & & $<0.001$ \\
\hline Ever smoked $(n=2093)$ & $2051(26)$ & 4799 (19) & $1572(16)$ & $227(19)$ & \\
\hline Never smoked $(n=35490)$ & $5965(74)$ & $20517(81)$ & $8063(84)$ & $945(81)$ & \\
\hline Missing $(n=413)$ & 358 & 52 & 3 & 0 & \\
\hline Socio-occupational status $(n=41733)$ & & & & & $<0.001$ \\
\hline High $(n=23171)$ & $3881(50)$ & $13265(56)$ & $5426(59)$ & $599(55)$ & \\
\hline Middle $(n=15154)$ & $2923(38)$ & $8913(37)$ & $2973(33)$ & $345(32)$ & \\
\hline Low $(n=3408)$ & $914(12)$ & $1678(7)$ & $670(7)$ & $146(13)$ & \\
\hline Missing $(n=2819)$ & 656 & 1512 & 569 & 82 & \\
\hline Leisure-time exercise during pregnancy, min/we & $k(n=41820)$ & & & & $<0.001$ \\
\hline $0(n=24607)$ & $4821(62)$ & $13914(58)$ & $5193(57)$ & $679(62)$ & \\
\hline $1-180(n=13479)$ & $2224(29)$ & $7898(33)$ & $3032(33)$ & $325(30)$ & \\
\hline $180+(n=3734)$ & $697(9)$ & $2086(9)$ & $859(9)$ & $92(8)$ & \\
\hline Missing ( $n=2732)$ & 632 & 1470 & 554 & 76 & \\
\hline Alcohol intake before pregnancy, units/week ( $n=$ & $44552)$ & & & & $<0.001$ \\
\hline $0(n=42,32)$ & $881(11)$ & $2301(9)$ & $898(9)$ & $152(13)$ & \\
\hline$>0-7(n=32168)$ & $5607(67)$ & $18610(73)$ & $7121(74)$ & $830(71)$ & \\
\hline$>7(n=8152)$ & $1886(23)$ & 4457 (18) & $1619(17)$ & $190(16)$ & \\
\hline Pre-pregnancy BMI before first live-birth $(n=40$ & & & & & $<0.001$ \\
\hline Underweight $(n=1827)$ & $384(5)$ & $970(4)$ & $397(5)$ & $66(6)$ & \\
\hline Normal weight $(\mathrm{n}=28330)$ & $5017(66)$ & $16287(70)$ & $6300(71)$ & $726(70)$ & \\
\hline Overweight $(n=7478)$ & 1435 (19) & 4340 (19) & $1523(17)$ & $180(17)$ & \\
\hline Obese $(n=3087)$ & $727(10)$ & $1680(7)$ & $608(7)$ & $72(7)$ & \\
\hline Missing $(n=3840)$ & 811 & 2091 & 810 & 128 & \\
\hline
\end{tabular}

Underweight, $<18.5 \mathrm{~kg} / \mathrm{m}^{2}$; normal weight, 18.5 to $<25 \mathrm{~kg} / \mathrm{m}^{2}$; overweight, 25 to $<30 \mathrm{~kg} / \mathrm{m}^{2}$; obese, $\geq 30 \mathrm{~kg} / \mathrm{m}^{2}$. Data are $n$ women (\% of parity group).

BMI, body mass index. 
Table 2 Live-birth pregnancies and risk (HRs and 95\% Cl) of hypertension by reproductive interval for women in the Danish National Birth Cohort

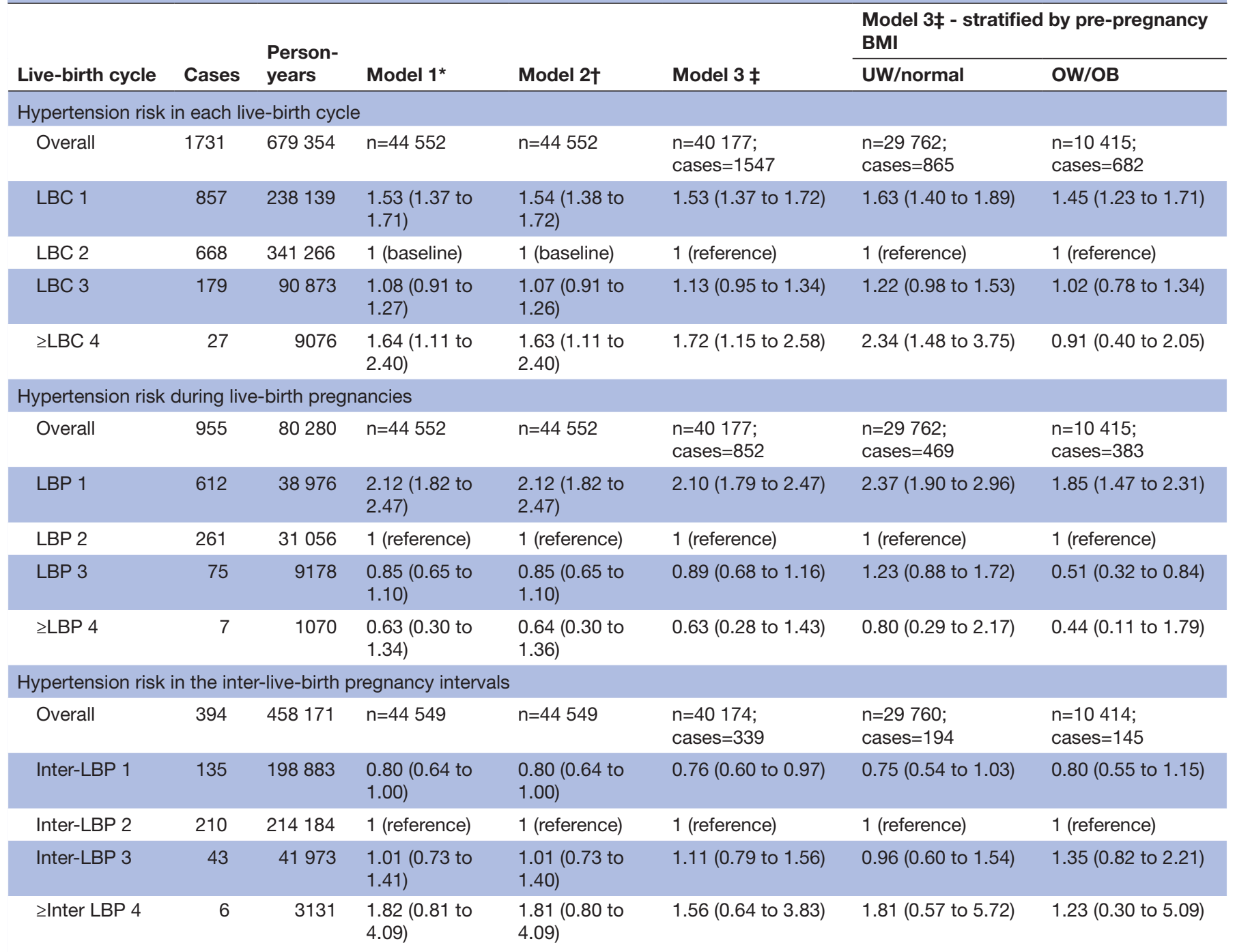

*Age as underlying time scale, adjusted for previous hypertension diagnosis in an inter-LBP interval for analysis of LBP intervals or previous hypertension diagnosis in a LBP interval for analysis of inter-LBP intervals

$\dagger$ Adjusted for $-^{*}$ and for induced abortions, spontaneous abortions and stillbirths (time-dependent variables)

$\ddagger$ Adjusted for $\dagger$ and for smoking status (ever/never), socio-occupational status, alcohol intake, exercise level and pre-pregnancy body mass index (underweight, normal-weight, overweight and obese)

BMI, body mass index; inter-LBP, inter-live-birth pregnancy; LBC, live-birth cycle; LBP, live-birth pregnancy;OB, obese; OW, overweight; UW, underweight.

association between the number of LBPs and risk of premenopausal hypertension in the entire follow-up period as hypothesised. This may be explained by our observation of increasing risk during LBPs and decreasing risk between LBPs as number of LBPs increased. The association between number of LBPs and risk of premenopausal CVD was similarly J-shaped, but somewhat weaker. Adjustment for pregnancy loss and pre-pregnancy BMI did not alter our findings. In contrast to our second hypothesis, associations among overweight/obese women were not stronger than among normal/underweight women. Thus, our findings suggest that increased number of LBPs may contribute to risk of premenopausal hypertension and CVD independent of BMI at the start of the reproductive period.

Few investigators have examined the associations between parity and risk of hypertension and CVD in women before menopause. However, parity may be more closely related to premenopausal risk than postmenopausal risk, as hormonal changes, ageing and other factors may become greater contributors to risk after menopause. ${ }^{23}$ Further, studies on postmenopausal risk include a study population free of hypertension or CVD at study enrolment in midlife, which limits comparability to our study. ${ }^{781011}$ 
Table 3 Live-birth pregnancies and risk (HR and 95\% Cl) of CVD by reproductive interval for women in the Danish National Birth Cohort

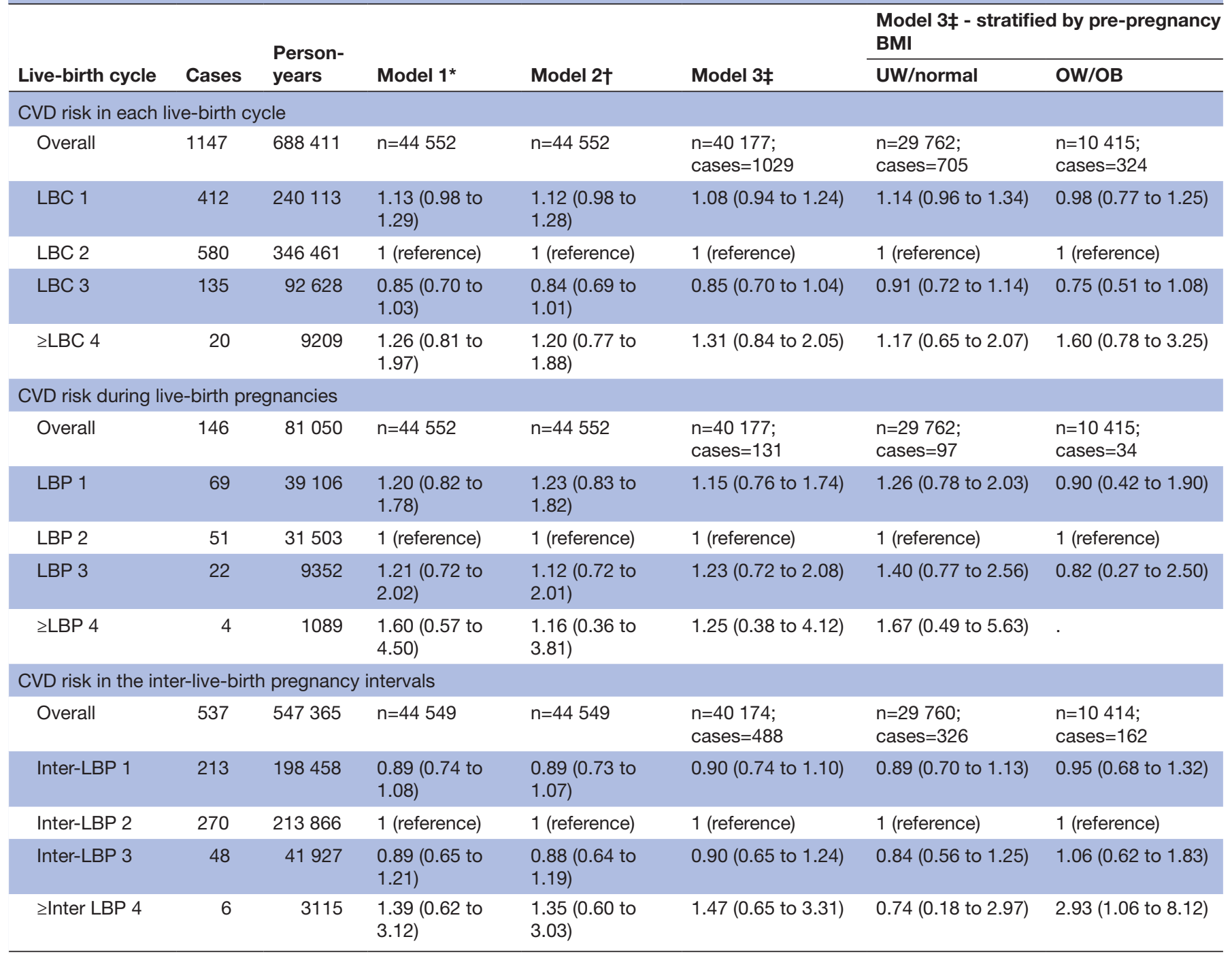

*Age as underlying time scale, adjusted for previous CVD diagnosis in an inter-LBP interval for analysis of LBP intervals or previous CVD diagnosis in a LBP interval for analysis of inter-LBP intervals

†Adjusted for * and for induced abortions, spontaneous abortions and stillbirths (time-dependent variables)

$\ddagger$ Adjusted for $\dagger$ and for smoking status (ever/never), socio-occupational status, alcohol intake, exercise level and pre-pregnancy body mass index (underweight, normal-weight, overweight and obese)

BMI, body mass index; CVD, cardiovascular disease;inter-LBP, inter-live-birth pregnancy; LBC, live-birth cycle; LBP, live-birth pregnancy; OB, obese; OW, overweight; UW, underweight.

Of the studies examining premenopausal risk, the results are generally consistent with our findings. Gold $e t$ $a l^{12}$ found that the risk of pregnancy-associated hypertension decreased after the first pregnancy. Given that the first pregnancy is a metabolic stressor, high-risk women are likely to be diagnosed with hypertension during this period. This may explain the increased risk we observed during the first LBP. Gaist $e t a l^{24}$ found that increasing parity was associated with a reduced risk of subarachnoid haemorrhage from first childbirth in primiparous women until an average age of 38.1 years. We also observed a decreased risk of CVD from the first LBC to the third; however, the risk increased again in the fourth and subsequent LBCs. This may be because we included a variety of CVD diagnoses in our outcome.

Pregnancy loss may increase women's risk of hypertension and CVD either by pathological processes from the pregnancy loss or by common underlying mechanisms of pregnancy itself. ${ }^{25}{ }^{26}$ We adjusted for pregnancy loss, but our findings did not change after adjustment, even though women with more LBPs at the end of their reproductive period tended to have more induced and spontaneous abortions.

Obesity may affect a woman's fertility ${ }^{27}$ and the metabolic changes during pregnancy. ${ }^{1528}$ However, adjustment for pre-pregnancy BMI category did not alter our results 
and we did not find any interactions by pre-pregnancy BMI category, except in risk of hypertension during LBPs. Although we had a large sample size, power for these analyses was low, which may have resulted in uncertain estimates. Still, the trend in our estimates did not support our hypothesised stronger association between LBPs and risk of hypertension and CVD in overweight/ obese women than normal/underweight. Also, obesity is a strong risk factor for hypertension and CVD in itself; thus, in overweight/obese women it may be difficult to ascertain variances in risks with additional LBPs.

A limitation of the study was that we had to assume that socio-occupational and lifestyle information obtained in the DNBC were constant throughout the reproductive period, as this information was only available for the first LBP. Therefore, we cannot rule out residual confounding due to changes in lifestyle factors related to child-rearing. ${ }^{11} 29$ Nevertheless, we focused on parous women who were all exposed to child-rearing and studied premenopausal risks that may be more likely associated with maternal metabolic changes compared with postmenopausal risks. Further, lifetime duration of breastfeeding may be a modifier of the association between parity and CVD. ${ }^{30}$ However, we were unable to consider lifetime duration of breastfeeding, as these data were not available. Lifetime breastfeeding duration may also be considered an intermediate factor between LBPs and risks of hypertension and CVD and therefore not a covariate to include in the analyses. In addition, misclassification may have occurred for hypertension disorders of pregnancy, as a previous validation study has observed a positive predictive value (PPV) of $66.7 \%$ for gestational hypertension. ${ }^{31}$ However, we assumed such misclassification to be unrelated to the exposure, and therefore non-differential. For diagnoses outside pregnancy, a high validity has been observed with PPVs above 95\% for chronic hypertension and myocardial infarction in women.$^{32}$ Finally, women who participated in the DNBC had a higher socio-occupational status and might be healthier than women in the overall Danish population, ${ }^{33}$ which limits the generalisability of our findings.

The primary strength of our study is our use of registry data linked to data from the DNBC. The combination of these data made it possible to develop a comprehensive reproductive record for each woman that eliminated self-reported information on reproductive events and included detailed information on socio-occupational, behavioural and anthropometric information not available in the registers. The reproductive record for each woman enabled us to consider premenopausal risks of hypertension and CVD during and between LBPs beginning from the start of the reproductive period to midlife while considering the women's pregnancy losses. Using register data reduced potential selection biases, as there was minimal loss to follow-up. Further, the large size of the cohort allowed us to restrict our study population to first-time mothers in the cohort and, thus, reduced selection bias. As observed in our sensitivity analysis, women who were parous at enrolment into DNBC had a different risk of hypertension and CVD during their first LBP than women who were nulliparous at enrolment. We saw this as a selection problem possibly due to fewer complications during the first LBP in parous women, who may also be healthier and more fertile, as they had become pregnant again. Finally, we studied premenopausal risks which may be more likely be related to maternal metabolic changes than postmenopausal risk of hypertension and CVD.

In conclusion, we found a J-shaped association between number of LBPs and risk of hypertension in a large sample of premenopausal women. Our results suggest that as number of LBPs increases, risk of hypertension decreases during pregnancies and increases between pregnancies. Findings did not vary after adjustment for induced abortions, spontaneous abortions and stillbirths, and may not be necessary to control for in future studies on parity. Moreover, adjusting for pre-pregnancy BMI category did not change the findings and women who were overweight/obese women before their first LBP did not have greater risks compared with women who were normal/ underweight. In premenopausal women, healthcare providers should consider the number of LBPs and the difference in risks of hypertension and CVD throughout the reproductive period in addition to the traditional risk factors for these diseases. Future work should focus on reproductive and cardiovascular factors during pregnancy that could reveal a biological basis for the observed associations between LBPs and premenopausal risk of hypertension and CVD.

Contributors HK, EAN, KMR and SXC designed research; SXC, HS and HK analysed data; SXC drafted the manuscript; HK, KMR, EAN, JLF and HS interpreted results and revised the manuscript; SXC and HK have primary responsibility for final content. All authors approved the final version for publication.

Funding The Danish National Birth Cohort was established with a significant grant from the Danish National Research Foundation. Additional support was obtained from the Danish Regional Committees, the Pharmacy Foundation, the Egmont Foundation, the March of Dimes Birth Defects Foundation, the Health Foundation and other minor grants. Helene Kirkegaard received a grant from the Danish Heart foundation grant number 14-R97-A5163. The Danish Heart foundation had no involvement in the design and conduct of the study, management, analysis and interpretation of the data or preparation, review or approval of the manuscript.

Competing interests None declared.

Patient consent for publication Not required.

Provenance and peer review Not commissioned; externally peer reviewed.

Data availability statement The data that provide the findings of this study are available from the Danish National Birth Cohort, but restrictions apply to the availability of these data, which were used under license for the current study, and so are not publicly available. Therefore, data described in the manuscript, code book, and analytic code will not be made available. All requests for data from the Danish National Birth Cohort must include a short protocol with a specific research question and a plan for analysis. More information can be found on www.dnbc.dk

Open access This is an open access article distributed in accordance with the Creative Commons Attribution Non Commercial (CC BY-NC 4.0) license, which permits others to distribute, remix, adapt, build upon this work non-commercially, and license their derivative works on different terms, provided the original work is properly cited, appropriate credit is given, any changes made indicated, and the use is non-commercial. See: http://creativecommons.org/licenses/by-nc/4.0/. 


\section{REFERENCES}

1. World Health Organ. Cardiovascular diseases (CVDs), 2015. Available: http://www.who.int/mediacentre/factsheets/fs317/en/ [Accessed 28 May 2015].

2. Cunningham FG, Williams JW. Williams obstetrics. 22nd ed. New York: McGraw-Hill Professional, 2005.

3. Gilmore LA, Klempel-Donchenko M, Redman LM. Pregnancy as a window to future health: excessive gestational weight gain and obesity. Semin Perinatol 2015;39:296-303.

4. Gongora MC, Wenger NK. Cardiovascular complications of pregnancy. Int J Mol Sci 2015;16:23905-28.

5. Wolff B, Völzke H, Robinson D, et al. Relation of parity with common carotid intima-media thickness among women of the study of health in Pomerania. Stroke 2005;36:938-43.

6. Jung SY, Bae HJ, Park BJ, et al. Parity and risk of hemorrhagic strokes. Neurology 2010;74:1424-9.

7. Parikh NI, Cnattingius S, Dickman PW, et al. Parity and risk of laterlife maternal cardiovascular disease. Am Heart J 2010;159:215-21.

8. Humphries $\mathrm{KH}$, Westendorp IC, Bots ML, et al. Parity and carotid artery atherosclerosis in elderly women: the Rotterdam study. Stroke 2001;32:2259-64.

9. Skilton MR, Sérusclat A, Begg LM, et al. Parity and carotid atherosclerosis in men and women: insights into the roles of childbearing and child-rearing. Stroke 2009;40:1152-7.

10. Zhang X, Shu X-O, Gao Y-T, et al. Pregnancy, childrearing, and risk of stroke in Chinese women. Stroke 2009;40:2680-4.

11. Lawlor DA, Emberson JR, Ebrahim S, et al. Is the association between parity and coronary heart disease due to biological effects of pregnancy or adverse lifestyle risk factors associated with childrearing? findings from the British women's heart and health study and the British regional heart study. Circulation 2003;107:1260-4

12. Gold RA, Gold KR, Schilling MF, et al. Effect of age, parity, and race on the incidence of pregnancy associated hypertension and eclampsia in the United States. Pregnancy Hypertens 2014;4:46-53.

13. Jang $M$, Lee $Y$, Choi J, et al. Association between parity and blood pressure in Korean women: Korean National health and nutrition examination survey, 2010-2012. Korean J Fam Med 2015;36:341-8.

14. Kotsis V, Stabouli S, Bouldin M, et al. Impact of obesity on 24hour ambulatory blood pressure and hypertension. Hypertension 2005;45:602-7.

15. Nelson SM, Matthews P, Poston L. Maternal metabolism and obesity: modifiable determinants of pregnancy outcome. Hum Reprod Update 2010;16:255-75.

16. Olsen J, Melbye M, Olsen SF, et al. The Danish National Birth Cohort--its background, structure and aim. Scand J Public Health 2001;29:300-7.
17. Knudsen LB, Olsen J. The Danish medical birth registry. Dan Med Bull 1998;45:320-3.

18. Bliddal M, Broe A, Pottegård A, et al. The Danish medical birth register. Eur J Epidemiol 2018;33:27-36.

19. Lynge E, Sandegaard JL, Rebolj M. The Danish national patient register. Scand J Public Health 2011;39(7 Suppl):30-3.

20. Tølbøll Blenstrup L, Knudsen LB. Danish registers on aspects of reproduction. Scand J Public Health 2011;39(7_suppl):79-82.

21. Nohr EA, Bech BH, Davies MJ, et al. Prepregnancy obesity and fetal death: a study within the Danish national birth cohort. Obstet Gynecol 2005;106:250-9.

22. World Health Organ. Maternal mortality ratio (per 100000 live births), 2014. Available: http://www.who.int/healthinfo/statistics/ indmaternalmortality/en/ [Accessed 28 May 2015].

23. Merz AA, Cheng S. Sex differences in cardiovascular ageing. Heart 2016;102:825-31.

24. Gaist D, Pedersen L, Cnattingius $S$, et al. Parity and risk of subarachnoid hemorrhage in women: a nested case-control study based on national Swedish registries. Stroke 2004;35:28-32.

25. Ranthe MF, Andersen EAW, Wohlfahrt J, et al. Pregnancy loss and later risk of atherosclerotic disease. Circulation 2013;127:1775-82.

26. Peters SAE, Yang L, Guo Y, et al. Pregnancy, pregnancy loss, and the risk of cardiovascular disease in Chinese women: findings from the China Kadoorie Biobank. BMC Med 2017;15:148.

27. Wise LA, Rothman KJ, Mikkelsen EM, et al. An Internet-based prospective study of body size and time-to-pregnancy. Hum Reprod 2010;25:253-64.

28. Gunderson EP, Abrams B, Selvin S. Does the pattern of postpartum weight change differ according to pregravid body size? Int $J$ Obes Relat Metab Disord 2001;25:853-62.

29. Magnus MC, lliodromiti S, Lawlor DA, et al. Number of offspring and cardiovascular disease risk in men and women: the role of shared lifestyle characteristics. Epidemiology 2017;28:880-8.

30. Klingberg S, Brekke HK, Winkvist A, et al. Parity, weight change, and maternal risk of cardiovascular events. Am J Obstet Gynecol $2017 ; 216$

31. Klemmensen AK, Olsen SF, Østerdal ML, et al. Validity of preeclampsia-related diagnoses recorded in a national Hospital registry and in a postpartum interview of the women. Am J Epidemio 2007;166:117-24.

32. Sundbøll J, Adelborg K, Munch T, et al. Positive predictive value of cardiovascular diagnoses in the Danish national patient registry: a validation study. BMJ Open 2016;6:e012832

33. Jacobsen TN, Nohr EA, Frydenberg M. Selection by socioeconomic factors into the Danish national birth cohort. Eur J Epidemiol 2010;25:349-55. 